\title{
QTc Dynamics Following Cardioversion for Persistent Atrial Fibrillation
}

\author{
Arwa Younis ${ }^{1}$, Nofrat Nehoray ${ }^{2}$, Michael Glikson ${ }^{3}$, Eyal Nof ${ }^{4}$, Michael Berger ${ }^{2}$, Wojciech \\ Zareba $^{5}$, Ilan Goldenberg ${ }^{5}$, and Roy Beinart ${ }^{4}$ \\ ${ }^{1}$ University of Rochester Medical Center \\ ${ }^{2}$ Sheba Medical Center at Tel Hashomer \\ ${ }^{3}$ Chaim Sheba Medical Center \\ ${ }^{4}$ Sheba Medical Center \\ ${ }^{5}$ University of Rochester
}

August 27, 2020

\begin{abstract}
Introduction - We aimed to assess changes in QTc over time following cardioversion (CV) for persistent atrial fibrillation (AF), and to compare the benefit of using continuous Holter monitoring vs. conventional follow-up. Methods - The study population comprised 90 patients admitted to our center for elective CV due to persistent AF who were prospectively enrolled from July 2017 through August 2018. All patients underwent 7-day Holter started prior to CV. Baseline QTc was defined as median QTc during first hour post CV. The primary endpoint was QTc prolongation defined as QTc [?]500ms, or [?]10\% increase (if baseline QTc was $>480 \mathrm{~ms}$ ). Conventional monitoring was defined as an ECG recording 2-hours post CV. McNemar test was used for comparison. Results - Mean age was $67 \pm 11$ years and $61 \%$ were male. Median baseline QTc was 452msec (IQ range: $431-479$ $\mathrm{msec}$ ) as compared with a maximal median QTc of 474msec (IQ range: 433-527 msec; p $<0.001$ for the change in QTc from baseline). Peak median QTc occurred 44-hours post CV. The primary endpoint was met in 3 patients (3\%) using conventional monitoring, compared with 39 new patients $(43 \%)$ using Holter $(\mathrm{p}<0.001$ for comparison). The Holter monitoring was superior to conventional monitoring in detecting clinically significant QTc prolongation $(\mathrm{OR}=13 ; \mathrm{p}<0.001)$. Conclusion $-\mathrm{CV}$ of patients with persistent AF may be associated with increased transient risk of QTc prolongation. Peak median QTc occurs during end of second day following CV and prolonged ECG monitoring provides superior detection of significant QTc prolongation compared with conventional monitoring
\end{abstract}

\section{Introduction}

Atrial fibrillation (AF) is the most common sustained arrhythmia worldwide and represents a major burden to health care systems. ${ }^{1}$

Rhythm control is the preferred strategy in patients with symptomatic AF. Electrical cardio-version (ECV) and pharmacological cardio-version (PCV) are widely used in order to restore sinus rhythm (SR). ECV is more effective than $\mathrm{PCV}$, particularly in persistent $\mathrm{AF}^{2}$, and while proven to be a safe, in some cases it could lead to serious adverse events, including QT prolongation and Torsade de pointes. ${ }^{3,4}$

It has been suggested that persistent AF induces ventricular repolarization remodeling leading to transient QT prolongation following ECV. ${ }^{5}$ This might increase the risk for Torsades de pointes, especially when other QT prolonging conditions exists. ${ }^{4}$ Furthermore, the European Heart Society and the American Heart Society guidelines recommend the initiation of antiarrhythmic drug therapy 1-3 days before electrical cardioversion to promote sustainable cardioversion. ${ }^{6,7}$ In fact, many of these drugs have a potential of further QTc prolongation, increasing the risk even more. ${ }^{8}$ Currently, there are no guidelines specifying the time needed to 
monitor patients following CV. The common practice is to watch the patients for 1-2 hours following CV, and thereafter to discharge them.

We hypothesized that patients with persistent atrial fibrillation on antiarrhythmic treatment might develop significant QTc prolongation following CV during long-term monitoring. Therefore, in this prospective clinical study we aimed to 1) assess changes in QTc following cardioversion and to identify the time to maximal QTc prolongation, 2) to compare the current standard of care to 7-days Holter monitoring, and 3) to identify clinical predictors for clinically significant QTc prolongation.

\section{Methods}

\section{Study Patients}

From July 09, 2017 through August 27, 2018, we prospectively enrolled consecutive patients with persistent AF who were admitted to the cardiology ward at the Chaim Sheba Medical Center (Tel Hashomer, Israel) for elective electric cardioversion. Our study inclusion criteria were: 1) age [?] 18 years old, 2) symptomatic persistent AF/AFL (atrial fibrillation or atrial flutter), 3) baseline QTc $>300 \mathrm{msec}, 4$ ) no contraindication for ECV, and 5) no contraindication for anticoagulation. Study exclusion criteria included: 1) recent initiation of medication that is well known to prolong QTc, 2) recent increase in dose of potentially prolonging medication (other than the medication desired for the CV), 3) pregnancy, 4) patients with congenital long QT syndrome, 5) baseline QTc $>500 \mathrm{msec}$, and 6) history of Torsade de pointes. Out of 136 consecutive patients, 100 patients agreed to participate in the study. All enrolled patients were connected to a 7-day 3-lead Holter prior to CV (in order to monitor QTc during AF)(Figure 1).

All patients underwent transesophageal echocardiography (TEE) if effective anticoagulation status was not confirmed or upon physician discretion. In addition, all underwent transthoracic echocardiography (TTE) within three months prior to the study enrollment, or on the day of CV. Of note, after connecting patients to Holter, ten patients (10\%) were withdrawn from the study due to left atrial appendage thrombus or severe swirling in TEE (4 patients), Holter malfunction (3), failure to cardiovert to sinus rhythm (2) and a patient who spontaneously converted to sinus rhythm following TEE, who was mistakenly discharged without further Holter monitoring. All remaining 90 patients were successfully CV to sinus rhythm and were monitored according to the study protocol using the Holter for 7 days.

The protocol was approved by the institutional review board of our center. All patients provided written informed consent. The primary hypothesis was that signifcant QTc prolongation can occur following ECV during 7 days of prolonged ECG monitoring, and that the use of Holter would be superior to the conventional protocol in detecting potentially clinically significant QTc prolongation.

\section{Medication and Electrical CV}

All patients were on antiarrhythmic drugs at baseline (Amiodarone $200 \mathrm{mg}$ once daily, Sotalol 80-120 mg twice daily, Flecainide $100 \mathrm{mg}$ twice daily, or Propafenone150-225 mg three times daily). After light sedation with Midazolam (up to a maximum of $5 \mathrm{mg}$, based on the patient weight), all patients underwent ECV with 200 Joules using a biphasic defibrillator patches. If sinus rhythm was not achieved and upon discretion of the treating physician, antiarrhythmic drugs were given for several 4-12 hours (Amiodarone 900-1200 $\mathrm{mg}$, Flecainide $400 \mathrm{mg}$, Propafenone $450 \mathrm{mg}$ ). After several hours (12 hours for Amiodarone, 4 hours for Flecainide and Propafenone) an ECG was performed. If the patient converted to SR, then the time of the CV was updated (based on the Holter results), and the patient was monitored for two hours post CV (conventional monitoring), thereafter discharged with Holter monitoring. If the patient was still in AF, ECV shock was re-delivered using the same sedation and shock protocol as described above. After achieving SR, patients were monitored for 2 hours (conventional monitoring) and thereafter discharged home with Holter monitoring (eFigure A in the online supplementary). Patients were seen in office for clinical follow-up at 1-3 months following CV.

\section{QTc measurement}


The methods of computerized QT analysis and their reproducibility have been described in detail previously. ${ }^{9,10}$ For this study we used a 3 lead Lifecard CF (OSI systems) Holter and data were analyzed using the Spacelab Pathfinder SL software. The Bazett formula was used to obtain heart rate-corrected values of parameters of QTc interval. Mean QTc was given hourly. All measurements were adjudicated by electrophysiology fellow (AY) and by internal Emergency Medicine resident (NN). If a change between two consecutive measurement $[(\mathrm{QTc} \text { second }-\mathrm{QTc} \text { first }) / \mathrm{QTc} \text { first }]^{*} 100$ was $>10 \%$, a manual validation/correction was performed. Furthermore, manual adjustment was made for patients with pacemaker during pacing periods. In order to minimize the errors that may occur during short tachy-arrhythmic events, or secondary to diurnal variability, the median of every 4 hours values was used. Accordingly, each patient had 6 measurement per day. Examples are listed in the online data supplement, eFigure B. In order to validate the results further, 10 random Holter strips were printed and evaluated blindly by a senior electrophysiologist (RB), and a fellow (AY), and the results were compared with the computerized results. The matching rate was $99.7 \%$. Examples are listed in the online data supplement, eFigures C-D.

\section{Definitions and endpoints}

Baseline QTc was defined as the median QTc during the first hour post CV. Conventional monitoring was defined as the Holter tracing at the second hour following CV. Holter monitoring was defined as tracings starting 3 hours following CV throughout the end of the monitoring period. The primary endpoint was clinically significant QTc prolongation defined as; 1) new prolongation of QTc [?]500 ms (if baseline QTc was $<480 \mathrm{~ms}$ ) or 2) prolongation of QTc [?]10\% if baseline QTc was $>480 \mathrm{~ms}$.

\section{Statistical analysis}

Continuous variables are expressed as mean \pm SD. Categorical data are summarized as frequencies and percentages. Median QTc baseline (first hour post CV), median QTc during the second hour (conventional monitoring), and the median QTc every 4 consecutive hours (Holter monitoring) were displaced in a graph with 25\%-75\% confidence interval, where the $\mathrm{Y}$ axis is the QTc, and $\mathrm{X}$ axis is time from CV.

We included 18 potential clinical, electrocardiographic, echocardiographic and laboratory binary risk factors for QTc prolongation (online supplemental eTable A). Numeric variables were made binary by the use of cut points with the goal of finding a simple, easily implemented predictors to be derived from them. Thresholds for categorization of numeric variables were based on the mean value. Univariate relationships between candidate covariates and a further event were assessed by t tests ( 2 for binary responses). The covariates with values of $\mathrm{P}<0.10$ were further evaluated by carrying out a best-subset regression analysis, examining the models created from all possible combinations of predictor variables, and using a penalty of 3.84 on the likelihood ratio 2 value for any additional factor included (corresponds to a $\mathrm{P}$ of $5 \%$ for a 1 -df 2 test). Model selection was repeated after unselected factors were dropped, one at a time, to minimize the effects of missing data.

Detection rates were calculated as a fraction of all patients who had received 7-day Holter monitoring. The cumulative probability of AF was displayed according to the Kaplan-Meier method. The differences between detection rates for different monitoring intervals were tested using McNemar's test as appropriate. All statistical tests were two-sided, a p-value of $<0.05$ was considered statistically significant. Analyses were carried out with SAS software (version 9.4, SAS institute, Cary, North Carolina).

\section{Results}

The baseline clinical characteristics of study patients are shown in Table 1 . The mean age of the study cohort was $67 \pm 11$ years, 55 patients $(61 \%)$ were male. The mean $\mathrm{CHA}_{2} \mathrm{DS}_{2} \mathrm{VASc}$ was $3.5 \pm 1.5$. Baseline mean heart rate in $\mathrm{AF}$ was $80 \pm 20 \mathrm{bpm}$. In 7 patients (8\%), CV was performed due to persistent atrial flutter. Most patients had hypertension $60(67 \%)$ and were likely to receive novel oral anti-coagulants 66 (73\%). Amiodarone was the most common antiarrhythmic medication used prior to CV (60 patients, 67\%). Despite the fact that the vast majority of patients were on adequate anti-coagulation status, TEE was performed in $63(70 \%)$ patients prior to $\mathrm{CV}$, due to increased stroke risk in this population. 
Eleven patients did not convert to SR with the first electrical shock. Reloading of the same anti-arrhythmic medication, as described in the methods section, was performed in them (8 patients with Amiodarone and 3 with Flecainide). Among them, 8 patients (5 Amiodarone, 3 Flecainide) converted to SR with the second ECV. The remaining 3 patients, needed a third ECV with 360 Joules in order to convert to SR.

\section{QTc dynamics during long-term Holter recording}

Patients underwent long term Holter monitoring for an average of $6.2 \pm 1.2$ days. Median baseline QTc (defined as the median QTc during first hour after CV) was $452 \pm 52 \mathrm{msec}$ and was significantly lower than the median QTc during the continuous Holter monitoring (467 $\pm 47 \mathrm{msec}, \mathrm{p}=0.01$ for comparison). The maximal median QTc occurred 44 hours post CV and was $474 \pm 100 \mathrm{msec}(\mathrm{p}<0.001$ for comparison with baseline QTc). Following this peak, the QTc returned to the baseline QTc 132 hours post CV, with a median QTc was $458 \pm 70 \mathrm{msec}$ ( $\mathrm{p}=0.58$ for comparison with baseline QTc) Figure 2. The baseline clinical characteristics were similar between those who had significant prolongation within the 44 hours post CV and those who developed it after. A trend towards more Amiodarone use was seen in those who developed a later QTc prolongation ( $87 \%$ vs $63 \%, \mathrm{p}=0.07$ ).

\section{Independent predictors of QTc prolongation}

In best subset forward regression analysis only prior beta blocker usage (HR 3.5, 95\% CI 1.04-12.3, p=0.042) was found to be independent predictor for potentially clinically significant QTc prolongation. Baseline QTc [?] 450 was significant enough to enter the final model $(\mathrm{p}<0.10)$, however, in the final multiple regression model the p-value was not statistically significant (HR 2.2, 95\% CI 0.89-5.2, $\mathrm{p}=0.09$ ) Table 2.

During the two hours following CV (conventional monitoring), 3 (4\%) patients met the primary endpoint. Among them, two patients had median QTc [?] $500 \mathrm{msec}(564$ and $500 \mathrm{msec}$ ), and one had an increase of $11 \%$. With the use of the 7-day Holter, significant QTc prolongation was detected in 39 patients (45\%), significantly higher than the rate observed using the conventional monitoring period $(\mathrm{p}<0.001)$. Notably, among the 87 patients without clinically significant QTc prolongation detected using conventional 2-hour monitoring, sixteen patients (18\%) developed new QTc prolongation during the first day, 8 patients (11\%) during the second day, and the rest thereafter. Importantly, as shown in Table 3, more than half of the QTc prolongation were detected within the first 2 days. Notably, during the second day, 5 patients had QTc $>$ $550 \mathrm{msec}$ (baseline QTc was less than $480 \mathrm{msec}$ in all of them).

Detection rates for every single day, and cumulative detection rates over the monitoring period are depicted in Figure 3. There was a strong recognizable pattern of detection rates favoring the first 48 hours of the monitoring period.

Using McNemar test for comparison, the Holter monitoring was superior to conventional monitoring in detecting the PE with an OR of 13; 95\%CI 5-65; $\mathrm{p}<0.001$.

\section{Clinical events and Death}

During the study period, one patient died on the $5^{\text {th }}$ day post CV. The cause of the death was congestive heart failure exacerbation and pulmonary edema. This patient did not have clinically significant QTc prolongation. None of our patients had Torsade de points or sustained ventricular arrhythmia. No other significant clinical events were reported.

\section{Discussion}

To the best of our knowledge this is the first observational prospective study that monitored closely the QTc interval following $\mathrm{CV}$ in patients with persistent $\mathrm{AF}$ using 7-day Holter monitoring. The main finding of this study include 1) significant QTc prolongation that was detected in $47 \%$ of patients following CV, 2) the maximal median QTc prolongation occurred during the second day (44 hours following CV), 3) there was a substantial increase in detection rates of clinically significant QTc prolongation using prolonged Holter monitoring compared with standard monitoring, and 4) chronic beta blocker use was the only predictor associated with significant QTc prolongation. 


\section{QTc prolongation following CV}

In our study, during prolonged monitoring using 7-day Holter, we were able to demonstrate a significant number of patients with new significant QTc prolongation following CV. In 32 patients (35\%), the median QTc reached new values [?]500 msec, and in 14 patients (16\%) it even reached QTc [?]550 msec (baseline QTc was $<480 \mathrm{msec}$ ). Our observations are in line with previous case series and small studies. ${ }^{11,12}$ Houltz and colleagues had previously reported QTc prolongation up to a mean of $672+-26 \mathrm{~ms}$ in patients that, in fact, developed Torsade de pointes. ${ }^{13}$ In another study Choy and colleagues reported that QTc was prolonged from $405+-55$ to $470+-67$ ms following CV. ${ }^{3}$ These reports, however, included a relatively small number of patients (less than 10 patients in average) or included patients who were treated with new antiarrhythmic drug, such as Sotalol or Dofetilide. Notably, in our study, neither initiation of a new antiarrhythmic medication nor an increase in dose occurred.

The exact mechanisms of QTc prolongation following CV remains unclear. Possible causes include: 1) bradycardia - several studies demonstrated that lower heart rates following CV may prolong the QTc interval ${ }^{14}$, 2) increased drug toxicity - changes in the neuro-hormonal status, such as change in sympathetic nervous activity and ANP were reported following $\mathrm{CV}^{15}$, and can potentially modulate the sensitivity to antiarrhythmic drugs, 3) abrupt slowing of heart rates, following termination of fast ventricular rates, that may lead to QTc prolongation ${ }^{16}$, 4) ventricular repolarization remodeling during atrial fibrillation - QTc during SR following CV was reported to be significantly and transiently prolonged (although of limited magnitude, the prolongation was substantial (approximately 15\%) in some individuals ${ }^{5}$ ), and 5) ion channels modification - in several previous studies ${ }^{17}$ an alterations in ion channels and several gene expressions in the atria and ventricle of patients with persistent $\mathrm{AF}$ were reported as leading to change in the QTc interval; In particular, the inward rectifier I(K1) channel, affecting the inward rectifier potassium current which regulates the terminal portion of phase 3 of the repolarization. ${ }^{18}$ Of note, in our study, we found that the magnitude of QTc prolongation was similar regardless of CV method, Midazolam use and regardless of the need to additional use/reloading of antiarrhythmic medication peri-cardioversion.

\section{Holter monitoring versus conventional monitoring}

Although the highest detection rate was observed during the first 24 hours following CV, a significant number of patients with QTc prolongation was detected thereafter. Furthermore, the maximum median QTc prolongation occurred during the second day (hour 44 post CV), which attenuated thereafter, returning to baseline QTc. Similar findings were reported in a study that tested the QT/RR relationship following ablation of the atrioventricular junction in patients with atrial fibrillation. It demonstrated that the highest change in QTc was documented on the second day [(516+-51ms on second day vs 468+-26ms baseline, $\mathrm{p}=0.02$; in group I) and ( $497+-37 \mathrm{~ms}$ on second day vs $458+-25 \mathrm{~ms}$ baseline, $\mathrm{p}=0.02$; in group II)], afterwards the QTc normalizes with no statistical difference observed from days 3 to 7 at all heart rates (14). In light of our findings there might be a need for further monitoring beyond several hours post CV in some individuals. In the past, and in accordance with this concept, the ACC/AHA/ESC 2006 Guidelines for the Management of Patients with AF recommended in hospital QT interval monitoring for 24-48 h following CV in patients receiving drugs that prolong the QT interval. However, in the most recent AHA/ACC and ESC guidelines, the above-mentioned recommendation were omitted, and the monitoring time following $\mathrm{CV}$ in patient on antiarrhythmic drugs became undefined. Hence, we believe that monitoring or repeated ECGs are essential during the 48 hours post CV in some patients with persistent AF on antiarrhythmic drugs.

\section{Predictors associated with significant QTc prolongation}

We found that chronic Beta blocker usage was associated with clinically significant QTc prolongation. Surprisingly, no other independent clinical or demographic characteristic was found to be associated with QTc prolongation. Previous studies have demonstrated controversy regarding the effect of beta blocker on the QT interval. Studies in patients with long QT demonstrated that at faster heart rates, beta-blockers shortened the maximum QT interval and resulted in shorter QTc, whereas at slower heart rates beta-blockers lengthened the maximum QT interval and resulted in longer QTc. ${ }^{19}$ We believe that these results are consistent 
with our findings as the prolongation of the QT interval was most likely to occur during relative bradycardia post CV. Furthermore, in patients with AF, Beta blocker medication may be a marker of a more resistant or more progressive AF disease with rapid ventricular response, resulting in the need for treatment with Beta blockers for rate management. In our study, we failed to show association between previously reported risk factors (female gender, age, potassium level, and magnesium level ${ }^{20}$ ) and the subsequent risk for QTc prolongation. We believe this is mainly due to the small number of our cohort.

Our study has several limitations. The lack of control group plays an important role, yet we aimed to assess the benefit of further monitoring per patient, using the conventional monitoring period as our control measurement. Some of the patients had AF recurrence (whether transient or persistent) which may affect the accuracy of the QTc calculation made by the software (using the Bazzet formula which may overestimate the QTc during tachycardia), however, we educated all the results with delta of more than $10 \%$, and we used a 4 hour median to correct for short episodes of tachyarrhythmias or bradyarrhythmia's.

\section{Conclusion}

The prior use of Beta blockers in addition to the antiarrhythmic medication seems to increase the risk for significant QTc prolongation following CV. A transient QTc prolongation following CV is a common finding in patients with persistent $\mathrm{AF}$ on antiarrhythmic medication, yet these prolongations seems to be safe without the development of sustained ventricular arrhythmia. Patients at increased risk for QTc prolongation may benefit from prolonged monitoring following CV.

\section{References}

1. Colilla S, Crow A, Petkun W, Singer DE, Simon T, Liu X. Estimates of current and future incidence and prevalence of atrial fibrillation in the U.S. adult population. Am J Cardiol2013;112:1142-1147.

2. Van Gelder IC, Tuinenburg AE, Schoonderwoerd BS, Tieleman RG, Crijns HJ. Pharmacologic versus direct-current electrical cardioversion of atrial flutter and fibrillation. Am J Cardiol1999;84:147R-151R.

3. Choy AM, Darbar D, Dell'Orto S, Roden DM. Exaggerated QT prolongation after cardioversion of atrial fibrillation. J Am Coll Cardiol 1999;34:396-401.

4. Spearritt D. Torsades de pointes following cardioversion: case history and literature review. Aust Crit Care 2003;16:144-149.

5. Tan HL, Smits JP, Loef A, Tanck MW, Hardziyenka M, Campian ME. Electrocardiographic evidence of ventricular repolarization remodelling during atrial fibrillation. Europace 2008;10:99-104.

6. January CT, Wann LS, Alpert JS, Calkins H, Cigarroa JE, Cleveland JC, Jr., Conti JB, Ellinor PT, Ezekowitz MD, Field ME, Murray KT, Sacco RL, Stevenson WG, Tchou PJ, Tracy CM, Yancy CW, Members AATF. 2014 AHA/ACC/HRS guideline for the management of patients with atrial fibrillation: a report of the American College of Cardiology/American Heart Association Task Force on practice guidelines and the Heart Rhythm Society. Circulation 2014;130:e199-267.

7. Kirchhof P, Benussi S, Kotecha D, Ahlsson A, Atar D, Casadei B, Castella M, Diener HC, Heidbuchel H, Hendriks J, Hindricks G, Manolis AS, Oldgren J, Popescu BA, Schotten U, Van Putte B, Vardas P, Group ESCSD. 2016 ESC Guidelines for the management of atrial fibrillation developed in collaboration with EACTS. Eur Heart J2016;37:2893-2962.

8. Roden DM. Drug-induced prolongation of the QT interval. N Engl J Med 2004;350:1013-1022.

9. McLaughlin NB, Campbell RW, Murray A. Accuracy of four automatic QT measurement techniques in cardiac patients and healthy subjects. Heart 1996;76:422-426.

10. Savelieva I, Yi G, Guo X, Hnatkova K, Malik M. Agreement and reproducibility of automatic versus manual measurement of QT interval and QT dispersion. Am J Cardiol 1998;81:471-477. 
11. Cellarier G, Deharo JC, Chalvidan T, Gouvernet J, Peyre JP, Savon N, Djiane P. Prolonged QT interval and altered QT/RR relation early after radiofrequency ablation of the atrioventricular junction.Am J Cardiol 1999;83:1671-1674, A1677.

12. Faber TS, Zehender M, Van de Loo A, Hohnloser S, Just H. Torsade de pointes complicating drug treatment of low-malignant forms of arrhythmia: four cases reports. Clin Cardiol 1994;17:197-202.

13. Houltz B, Darpo B, Edvardsson N, Blomstrom P, Brachmann J, Crijns HJ, Jensen SM, Svernhage E, Vallin H, Swedberg K. Electrocardiographic and clinical predictors of torsades de pointes induced by almokalant infusion in patients with chronic atrial fibrillation or flutter: a prospective study. Pacing Clin Electrophysiol 1998;21:1044-1057.

14. Trinkley KE, Page RL, 2nd, Lien H, Yamanouye K, Tisdale JE. QT interval prolongation and the risk of torsades de pointes: essentials for clinicians. Curr Med Res Opin 2013;29:1719-1726.

15. Salerno DM, Katz A, Dunbar DN, Fjeldos-Sperbeck K. Serum electrolytes and catecholamines after cardioversion from ventricular tachycardia and atrial fibrillation. Pacing Clin Electrophysiol1993;16:18621871.

16. Viskin S. Post-tachycardia QT prolongation: maladjustment of the QT interval to the normal heart rate. Pacing Clin Electrophysiol 2003;26:659-661.

17. Nattel S, Maguy A, Le Bouter S, Yeh YH. Arrhythmogenic ion-channel remodeling in the heart: heart failure, myocardial infarction, and atrial fibrillation. Physiol Rev 2007;87:425-456.

18. Anumonwo JM, Lopatin AN. Cardiac strong inward rectifier potassium channels. J Mol Cell Cardiol 2010;48:45-54.

19. Bennett MT, Gula LJ, Klein GJ, Skanes AC, Yee R, Leong-Sit P, Chattha I, Sy R, Jones DL, Krahn AD. Effect of beta-blockers on QT dynamics in the long QT syndrome: measuring the benefit. Europace 2014;16:1847-1851.

20. Tisdale JE, Jaynes HA, Kingery JR, Mourad NA, Trujillo TN, Overholser BR, Kovacs RJ. Development and validation of a risk score to predict QT interval prolongation in hospitalized patients. Circ Cardiovasc Qual Outcomes 2013;6:479-487.

\section{Table 1 - Baseline Characteristics}

\begin{tabular}{llll}
\hline & Study Cohort (No.=90) & $\begin{array}{l}\text { Study Cohort } \\
\text { Significant QTc Prolongation } \\
\text { No (No.=48) }\end{array}$ & $\begin{array}{l}\text { Study Cohort } \\
\text { Significant QTc } \\
\text { Yes (No.42) }\end{array}$ \\
Male, \% & & $26(60)$ & $27(61)$ \\
Age, mean (SD) & $55(61)$ & $67 \pm 12$ & $67 \pm 11$ \\
BMI, mean (SD), kg/m2 & $67 \pm 11$ & $29 \pm 4$ & $30 \pm 5$ \\
Atrial flutter & $30 \pm 5$ & $5(11)$ & $2(4)$ \\
Heart Rate in AF, mean (SD), bpm & $7(8)$ & $78 \pm 21$ & $82 \pm 20$ \\
Ischemic Heart Disease, \% & $19(21)$ & $8(16)$ & $12(27)$ \\
CHF, \% & $30(36)$ & $12(29)$ & $18(43)$ \\
TEE, \% & $63(70)$ & $31(63)$ & $32(76)$ \\
EF, mean (SD), \% & $52 \pm 12$ & $54 \pm 12$ & $49 \pm 14$ \\
LA size, mean (SD), cm & $4.5 \pm 0.4$ & $23(48)$ & $4.4 \pm 1$ \\
Mitral regurgitation, \% & $56(62)$ & $7(15)$ & $33(79)$ \\
Mitral stenosis, \% & $14(15)$ & $6(14)$ & $7(17)$ \\
Aortic stenosis, \% & $12(13)$ & $34 \pm 8$ & $6(15)$ \\
SPAP, mean (SD), mmHg & $34 \pm 8$ & $9(19)$ & $35 \pm 7$ \\
Diabetes, \% & $25(28)$ & $29(67)$ & $16(36)$ \\
Hypertension, \% & $60(67)$ & & $30(68)$
\end{tabular}




$\begin{array}{llll}\text { Renal disease, } \% & 21(23) & 10(24) & 11(25) \\ \mathrm{CHA}_{2} \mathrm{DS}_{2} \text { VASc, mean (SD) } & 3.5 \pm 1.5 & 3.3 \pm 1.6 & 3.7 \pm 1.7 \\ \text { ICD/CRTD, \% } & 2(2) & \text { None } & 2(4) \\ \text { Pacemaker } & 11(12) & 5(11) & 6(15) \\ \text { ACE Inhibitor or ARB, } \% & 47(52) & 22(46) & 25(60) \\ \text { Aldosterone, \% } & 10(11) & 4(9) & 6(15) \\ \text { Beta-blocker, \% } & 67(74) & 30(63) & 37(88) \\ \text { Calcium channel blocker, } \% & 25(28) & 12(26) & 13(29) \\ \text { Digitalis, \% } & 6(7) & 2(4) & 4(9) \\ \text { Amiodarone, } \% & 60(67) & 30(63) & 30(69) \\ \text { Flecainide, \% } & 20(22) & 10(21) & 10(23) \\ \text { Propafenone, } \% & 5(6) & 4(9) & 1(2) \\ \text { Statins, \% } & 39(43) & 21(46) & 18(42) \\ \text { NOAC, \% } & 66(73) & 33(69) & 33(76) \\ \text { Warfarin, \% } & 24(27) & 14(30) & 10(23) \\ \text { Creatinine, mean }(\mathrm{SD}), \mathrm{mg} / \mathrm{dl} & 1 \pm 0.3 & 1 \pm 0.3 & 0.9 \pm 0.3 \\ \text { K, mean (SD), mg/dl } & 4.3 \pm 0.4 & 4.3 \pm 0.4 & 4.3 \pm 0.4 \\ \text { Na, mean (SD), mg/dl } & 140 \pm 4 & 140 \pm 3 & 140 \pm 2 \\ \text { Mg, mean (SD), mg/dl } & 2 \pm 0.4 & 2 \pm 0.2 & 2 \pm 0.4\end{array}$

$\mathrm{AF}=$ atrial fibrillation; $\mathrm{ACE}=$ angiotensin converting enzyme; $\mathrm{ARB}=$ angiotensin receptor blocker; $\mathrm{BMI}=$ body mass index; $\mathrm{CHF}=$ congestive heart failure $\mathrm{COPD}=$ chronic obstructive pulmonary disease; $\mathrm{CRTD}=$ cardiac resynchronization therapy with a defibrillator; $\mathrm{ICD}=$ implantable cardioverter defibrillator; $\mathrm{EF}=$ ejection fraction; $\mathrm{LA}=$ left atrial dimension; $\mathrm{NOAC}=$ novel oral anti-coagulant; $\mathrm{SPAP}=$ systolic pulmonic arterial pressure; $\mathrm{TEE}=$ trans-esophageal echocardiography.

Table 2 - Predictors for Clinically Significant QTc Prolongation 7-day Holter versus 2-hours conventional monitoring.

\begin{tabular}{ll}
\hline End point: & Hazard ratio
\end{tabular}

\begin{tabular}{ll}
\hline Clinically Significant QTc Prolongation (39 events) & Clinically Significant QTc Prolongation (39 events) \\
Beta Blocker & 3.5 \\
Median QTc [?] 450 during first hour post CV & 2.2 \\
\hline
\end{tabular}

$\mathrm{CV}=$ cardioversion

Table 3 - Rates of Clinically Significant QTc during Conventional Monitoring and during 7-day Holter.

\begin{tabular}{llll}
\hline & New Clinically Significant QTc Prolongation & Percentage of new events & No. of remaining patients witho \\
\hline Conventional & 3 & $3 \%$ & 87 \\
First Day & 16 & $18 \%$ & 71 \\
Second Day & 8 & $11 \%$ & 63 \\
Third Day & 7 & $11 \%$ & 56 \\
Fourth Day & 3 & $5 \%$ & 53 \\
Fifth Day & 4 & $8 \%$ & 49 \\
Sixth Day & 1 & $2 \%$ & 48 \\
Seventh Day & 0 & $0 \%$ & 48 \\
7 Days Holter & 39 & $43 \%$ & 51 \\
\hline
\end{tabular}




\section{Figure 1 - Study Flow Chart}

* Trans esophageal echocardiography (TEE) was performed in two third of the patients due to their high risk profile.

lead three was disconnected prior to the CV and remained disconnected thereafter.

Figure 2 - Median QTc obtained by Continuous 7 Day Holter following Elective Cardioversion (CV) for Persistent Atrial Fibrillation. Time Zero Represents the CV Time.

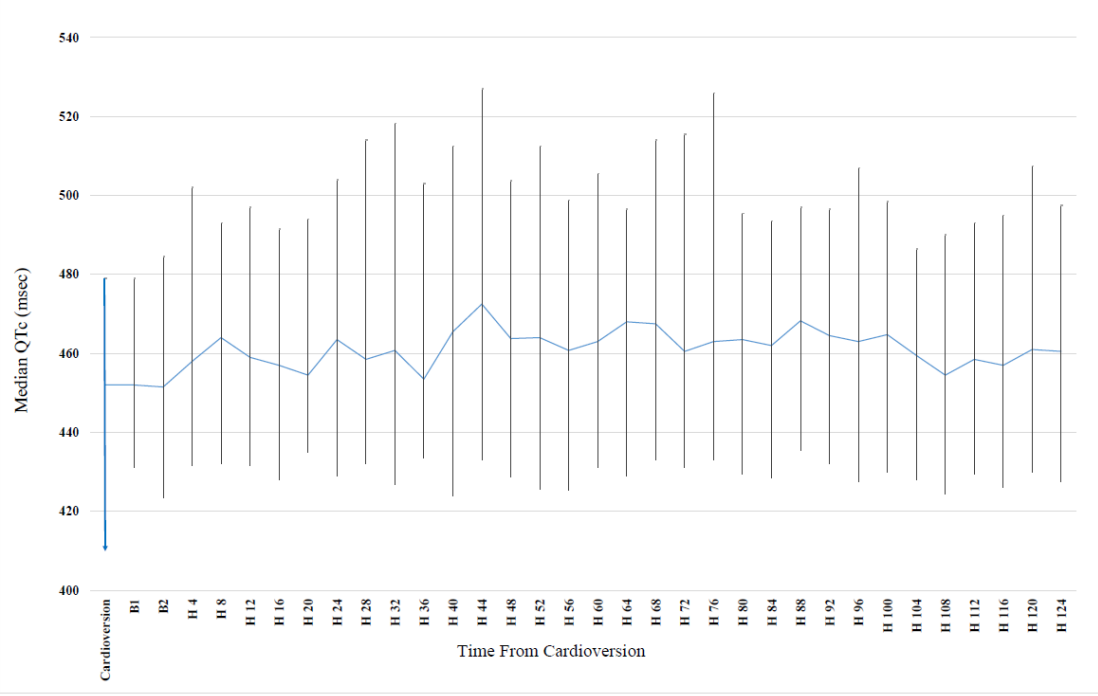

At time $132 \mathrm{~h}$ post CV, 33 (37\%) of the patients were no longer connected to the 7-day Holter (due to longer detection prior to $\mathrm{CV}$ ). The rate of patients connected to the Holter became lower every further hour beyond the $132 \mathrm{~h}$ post $\mathrm{CV}$, making it incorrect to calculate for means and draw conclusions from it. Therefore, the graph applies only until $128 \mathrm{~h}$ post $\mathrm{CV}$

Figure 3 - Daily/Conventional (columns) and Cumulative (line) Detection Rates of Potentially Dangerous QTc Prolongation Following Cardioversion during 7 days of Holter Monitoring. 


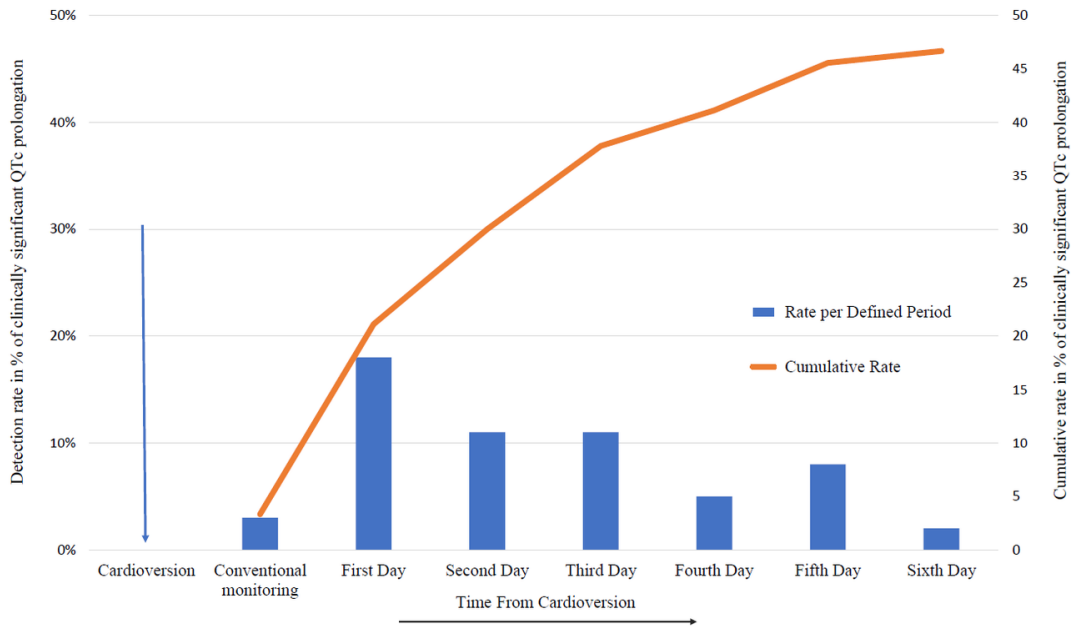




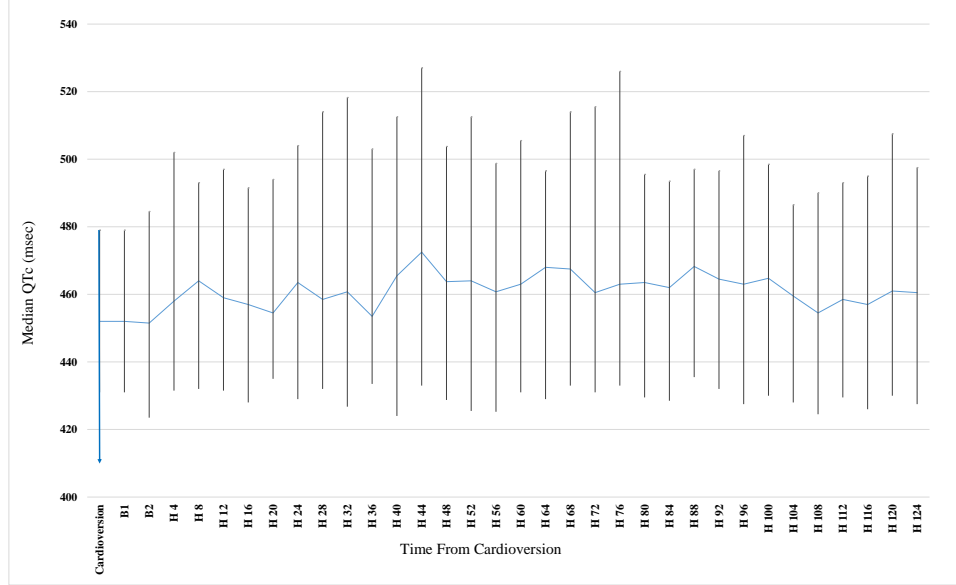




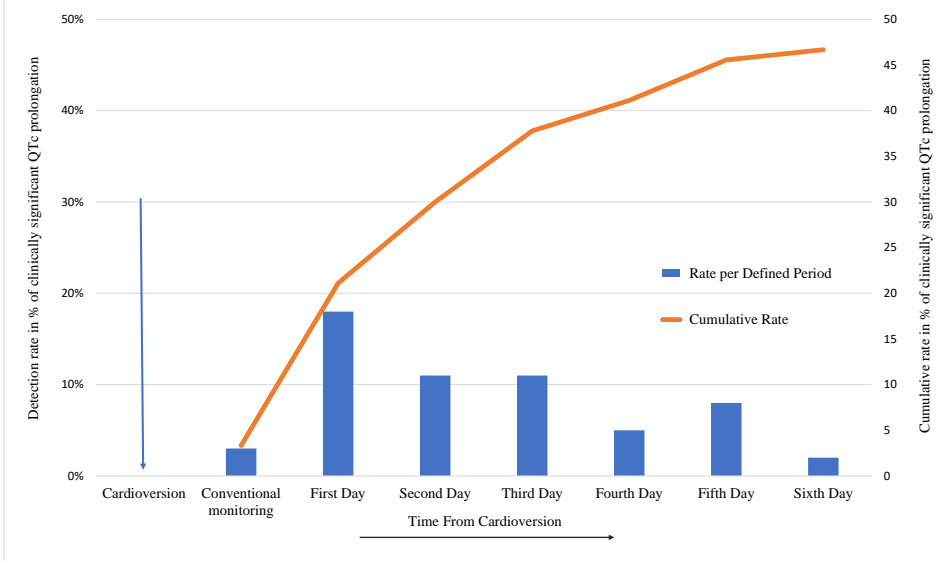

